\title{
Aumento na Prevalência de Diabete Melito Como Causa de Insuficiência Renal Crônica Dialítica - Análise de 20 Anos na Região Oeste do Paraná
}

\begin{abstract}
RESUMO
A nefropatia diabética (ND) é a principal causa de insuficiência renal crônica terminal (IRCT) nos países desenvolvidos. Objetivos: Observar se ocorreu aumento da prevalência de diabete melito (DM) como causa de IRCT nos últimos 20 anos e comparar a sobrevida em diálise dos diabéticos e não diabéticos. Métodos: Análise retrospectiva dos pacientes mantidos em diálise na região Oeste do Paraná no período de 1985 a 2005. A estimativa de sobrevida foi realizada pela Curva de Sobrevida de Kaplan-Meier. Resultados: Durante este período, foram admitidos em diálise 645 pacientes. Em 16,1\% deles o DM foi a causa da IRCT. Observou-se aumento na prevalência de DM como causa de IRCT. A sobrevida dos pacientes em diálise foi inferior nos diabéticos. Conclusões: A prevalência de DM como causa de IRCT aumentou progressivamente nos últimos 20 anos em nossa região. A sobrevida de pacientes diabéticos em diálise foi menor que a dos não-diabéticos. (Arq Bras Endocrinol Metab 2007;51/1:111-115)
\end{abstract}

Descritores: Doença renal crônica terminal; Incidência; Nefropatia diabética; Diabete melito

\section{ABSTRACT}

Increase in Prevalence of Diabetes Mellitus as a Cause of Dialytic End-Stage Renal Disease - Analysis of 20 Years in the West Region of Paraná.

Diabetic nephropathy (DN) is the main cause of chronic kidney disease (CKD) in developed countries. Objective: To observe if there was an increase in the prevalence of diabetes mellitus (DM) of CKD in the last 20 years and to analyze comparatively the survival on dialysis of diabetic and non-diabetic patients. Methods: Retrospective analysis of patients kept on dialysis in the west region of Paraná State in the period between 1985-2005. Survival analysis was performed using Kaplan-Meier Curves. Results: In the period, 645 patients were admitted to dialysis. In $16.1 \%$ DM was the cause of the CKD. It was observed a progressive increase in the prevalence of DM as a cause of CKD. Patient survival was lower in diabetics. Conclusions: The prevalence of DM as a cause of CKD increased in the last 20 years in our region. The survival rates were lower in diabetic than in non-diabetic patients. (Arq Bras Endocrinol Metab 2007;51/1:111-115)

Keywords: End-stage renal disease; Incidence; Diabetic nephropathy; Diabetes mellitus

DIABETE MELITO (DM) é a principal causa de insuficiência renal crônica terminal (IRCT) em países desenvolvidos. O número de pacientes diabéticos que anualmente vêm sendo incluídos em tratamento substitutivo da função renal tem aumentado nos últimos anos na maioria dos países, e é representada principalmente por diabéticos do tipo $2(1,2)$. Em revisão muito perspectivas

\author{
LUIS A.B. PERES \\ TIEMI MATSUO \\ VINICIUS D.A. DELFINO \\ Celeide P.A. Peres \\ José HenRIQue de Almeida Netto \\ HI K. ANN \\ MaURício T.A. Camargo \\ NORIS R.S. ROHDE \\ VANESSA F.M. USCOCOVICH
}

Universidade Estadual do Oeste do Paraná - UNIOESTE,

Universidade Estadual de

Londrina - UEL e Renalclín Oeste,

Cascavel, PR.

Recebido em 04/1 1/05

Revisado em 17/04/06 e 13/07/06

Aceito em 15/09/06 
Peres et al.

recente sobre o tema, Locatelli e cols. citam que o DM representa $45 \%$ dos casos novos de IRCT nos Estados Unidos, 36\% na Alemanha, 32\% no Japão e 15-25\% em vários outros países europeus e na Austrália (3). Oliveira e cols. relatam que atualmente no Brasil, e na América Latina como um todo, cerca de $15 \%$ dos pacientes em diálise são diabéticos (4). Não encontramos, no entanto, na literatura dados nacionais sobre a evolução da incidência de DM como causa de IRC dialítica.

A taxa de mortalidade dos pacientes diabéticos em diálise é sabidamente maior que a dos não-diabéticos. Os fatores associados a isso são decorrentes do envolvimento vascular destes pacientes no início da diálise devido a processo aterosclerótico (5). Co-morbidades agravam o prognóstico, dentre elas história prévia de infarto do miocárdio, acidente vascular cerebral e doença vascular periférica $(2,6)$.

Os objetivos do presente trabalho foram avaliar a prevalência de DM como causa de IRCT na região Oeste do Estado do Paraná nos últimos 20 anos, e comparar os dados dos diabéticos e não diabéticos em diálise, especialmente sobre quanto à sobrevida.

\section{CASUÍSTICA E MÉTOdOS}

Foram revisados os prontuários de todos os pacientes renais crônicos admitidos para tratamento dialítico crônico nos seis Hospitais da cidade de Cascavel, PR, os quais atendem a área de abrangência da 10a Regional de Saúde do Paraná, que compreende 25 municípios com população estimada em aproximadamente 500.000 habitantes, no período de março de 1985 a fevereiro de 2005 . No total, 645 pacientes renais crônicos iniciaram diálise neste período. Foram levantados os dados referentes a sexo, idade, raça, doença básica, data de início de terapia renal substitutiva, data e motivo da saída do programa dialítico. Foram comparados os dados de todos os pacientes cuja doença básica foi o DM, com aqueles cuja doença básica não foi o DM. Os critérios utilizados para definição de nefropatia diabética foram a presença de história de DM de longa data com evolução para IRCT. Definiu-se glomerulonefrite crônica (GNC) na presença de proteinúria e/ou hematúria com evolução para IRCT, documentada por biópsia renal prévia. Foi considerada hipertensão arterial como doença básica quando havia história de hipertensão arterial sistêmica com evolução progressiva para IRCT em indivíduos não portadores de DM ou GNC. Considerou-se doença básica indeterminada quando os pacientes chegavam em IRCT sem evidência clínica de doença básica. DM tipo 1 foi considerado quando o início da doença ocorreu na adolescência com tendência à cetoacidose. $\mathrm{O}$ diagnóstico de DM tipo 2 foi estabelecido de acordo com os seguintes critérios: início do DM após os 30 anos de idade, pacientes usualmente obesos, sem tendência à cetose durante condições de estresse severo (7). Todos os pacientes diabéticos foram encaminhados por endocrinologistas, que utilizaram como critério diagnóstico o WHO Study Group de 1985 (8). O período de 20 anos do estudo foi dividido em qüinqüênios, e foi calculada a prevalência de DM como causa de IRCT em cada um deles.

\section{Análise estatística}

Na comparação dos dados demográficos, foram utilizados os testes Mann-Whitney para variáveis contínuas e Qui-quadrado ou Exato de Fisher para variáveis categóricas, conforme necessário. Foram estimadas as sobrevidas dos portadores de DM e dos demais pacientes através da Curva de sobrevida de Kaplan-Meier, ajustada para sexo e idade. A comparação entre as curvas de sobrevida foi feita pelo teste de Log-rank. Foram censurados da análise da curva de sobrevida saídas de programa dialítico por transferência, transplante renal ou tempo de permanência inferior a trinta dias em tratamento.

\section{RESULTADOS}

No período de março de 1985 a fevereiro de 2005, 645 pacientes iniciaram tratamento dialítico em Cascavel, PR, sendo que o DM foi a causa atribuída da IRCT de 104 pacientes $(16,1 \%$ de todas as causas de IRCT), 88 $(85,5 \%)$ destes pacientes eram diabéticos tipo 2 . Em relação aos pacientes diabéticos, a idade média ao início da terapia renal substitutiva foi significativamente maior que a dos pacientes não-diabéticos $(54,6 \pm 12,4$ anos vs. 42,8 $\pm 15,7$ anos), $65,4 \%$ eram homens e $83,6 \%$ brancos. As outras causas de IRCT mais freqüentes foram hipertensão arterial $36,1 \%$ e glomerulonefrite crônica em $24,5 \%$ dos casos. Os dados demográficos dos pacientes estão representados na tabela 1 .

Quando dividimos o período de 20 anos em quatro qüinqüênios, observamos que a prevalência de DM como causa de IRCT foi $6,9 \%$ no primeiro ( 1985 a 1990), 10,4\% no segundo (1990 a 1995), 13,7\% no terceiro (1995 a 2000 ) e de $24,6 \%$ no último (2000 a 2005). Estes dados estão representados na figura 1. Os pacientes censurados somente o foram para a análise da curva de sobrevida.

Dos 104 pacientes diabéticos crônicos renais, três $(2,9 \%)$ foram transferidos para outro serviço, 10 $(9,7 \%)$ submetidos a transplante renal e $59(57,3 \%)$ foram a óbito no período referido. No momento da análise, 31 pacientes se encontravam em tratamento dialítico, 29 em hemodiálise e dois em diálise peritoneal ambulatorial contínua.

A figura 2 mostra as curvas de sobrevida de pacientes diabéticos e não diabéticos, ajustadas segundo sexo e idade. A sobrevida aos cinco anos foi, respectivamente, de $18,5 \%$ e $58,8 \%$ para pacientes com e sem $\mathrm{DM}$, diferença estatisticamente significante $(\mathrm{p}<0,05)$. 
Tabela 1. Dados demográficos dos pacientes em tratamento dialítico.

\begin{tabular}{lccc}
\hline Variáveis demográficas & $\begin{array}{c}\text { Diabéticos } \\
(\mathbf{N}=\mathbf{1 0 4})\end{array}$ & $\begin{array}{c}\text { Não Diabéticos } \\
\text { (N= 541) }\end{array}$ & Valor de p \\
Sexo masculino & $68(65,4 \%)$ & $321(59,3 \%)$ & $0,2481^{*}$ \\
Raça & $87(83,6 \%)$ & $443(81,9 \%)$ & $0,6661^{*}$ \\
Branca & $17(16,4 \%)$ & $98(18,1 \%)$ & $<0,001^{\dagger}$ \\
Não branca & $54,6 \pm 12,4(23-74)$ & $42,8 \pm 15,7(2-85)$ & $0,2738^{*}$ \\
Idade (média, em anos) & $20(19,2 \%)$ & $81(15,0 \%)$ & $<0,0001^{*}$ \\
Tipo de diálise & $84(80,8 \%)$ & $460(85,0 \%)$ & \\
Peritoneal & $10(9,6 \%)$ & $173(32,0 \%)$ & $<0$. \\
Hemodiálise & Transplantados & & \\
\hline
\end{tabular}

* Teste de qui-quadrado; ${ }^{\dagger}$ Teste de Mann-Whitney

Prevalência

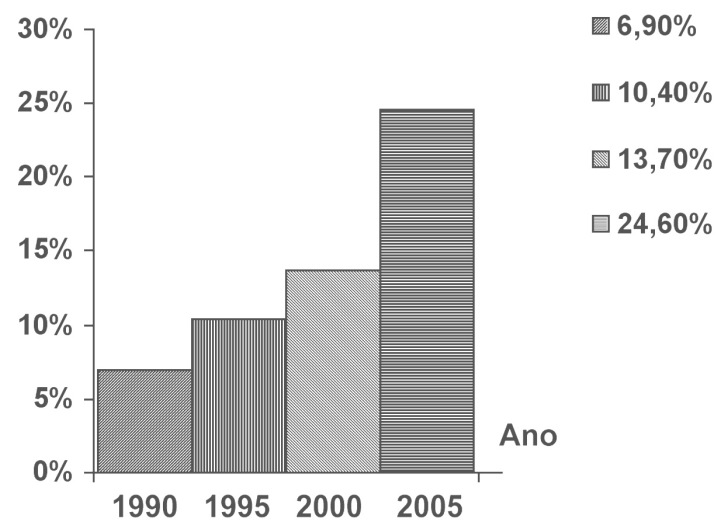

Figura 1. Prevalência de diabete melito como causa de IRCT nos últimos 20 anos, divididos em quatro qüinqüenios.

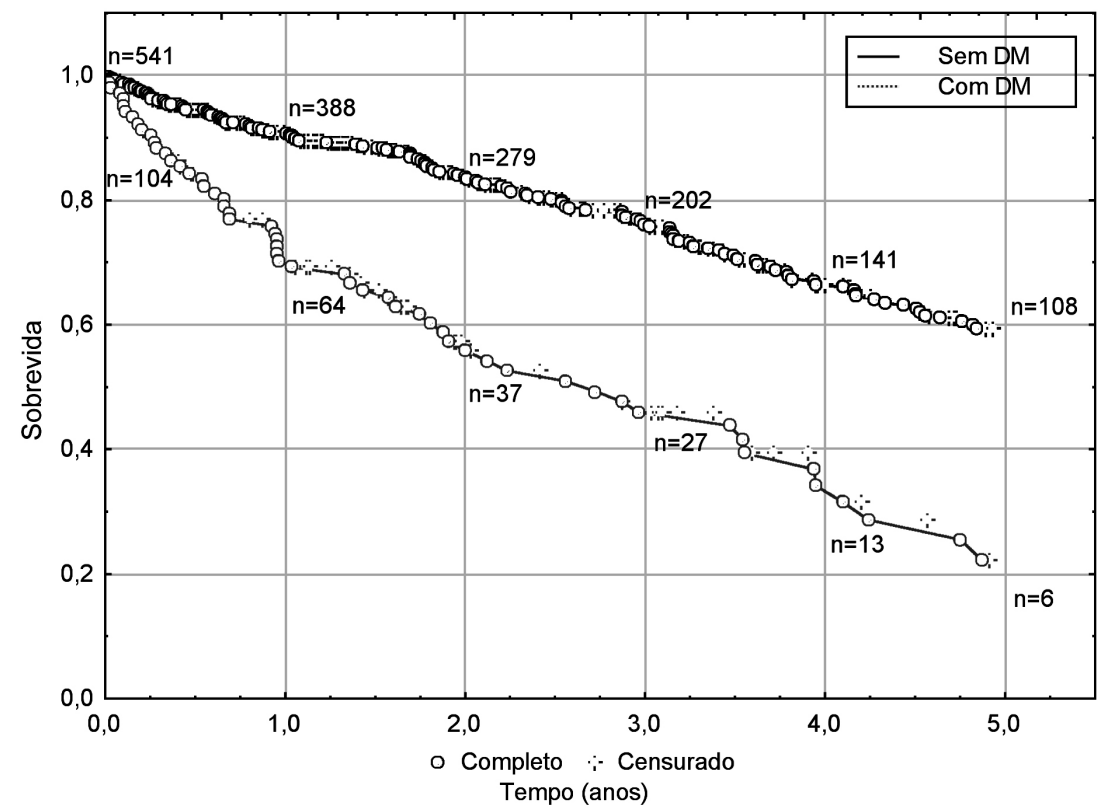

Figura 2. Curva de Sobrevida de Kaplan-Meier para pacientes renais crônicos em diálise com e sem diabete melito, ajustada para sexo e idade. 


\section{DISCUSSÃO}

O presente estudo demonstrou um aumento progressivo na prevalência de DM nos pacientes admitidos para tratamento dialítico crônico ao longo dos 20 anos de observação. A causa deste aumento da prevalência de pacientes diabéticos com IRCT no mundo é multifatorial. Uma das explicações para esse incremento na incidência de nefropatia diabética (ND) é o aumento na incidência de DM tipo 2 na população geral, decorrente da epidemia de obesidade. Nos Estados Unidos, $4 \%$ da população é diabética, sendo mais de $80 \%$ dos casos devido ao DM tipo 2 (9) A prevalência do DM tipo 2 na população adulta brasileira era de 7,6\% no início da década de 90, e estima-se que no ano de 2025 ocorra um aumento de $178 \%$ (10).

Estima-se que 20 a $45 \%$ dos pacientes diabéticos desenvolverão nefropatia ao final de 10-15 anos de observação (11-14). Em alguns grupos geneticamente susceptíveis como os índios Pima norte-americanos, taxas de nefropatia diabética tão altas quanto $42 \%$ após cinco anos do diagnóstico do diabetes tipo 2 são relatadas (15). A prevalência de DM tipo 2 é dez vezes superior à do DM tipo $\mathrm{l} \mathrm{e}$, por isto, observa-se uma maior prevalência de IRCT secundária ao DM tipo 2 (16), como observado no presente estudo. Este achado está de acordo com outros estudos nacionais (2).

Atualmente são reconhecidos alguns fatores de risco para o desenvolvimento da ND, são eles: predisposição genética (história de hipertensão e eventos cardiovasculares em parentes de primeiro grau), qualidade do controle glicêmico e lipídico, nível de pressão arterial e tabagismo (17).

Quando comparamos a sobrevida dos pacientes diabéticos com a dos não-diabéticos em programa dialítico, observamos que o diabético apresentou menor sobrevida. Dados semelhantes foram obtidos por outros centros do Brasil (2). A sobrevida aos cinco anos foi, respectivamente, de $18,5 \%$ e $58,8 \%$ para pacientes com e sem DM, diferença estatisticamente significante $(\mathrm{p}<0,05)$. A realidade é semelhante em países desenvolvidos: a sobrevida do diabético em diálise aos cinco anos na Alemanha é de aproximadamente 6\% (18). A taxa de mortalidade dos pacientes diabéticos em diálise é sabidamente maior que a dos não-diabéticos. Os fatores associados a isto são decorrentes do envolvimento vascular destes pacientes no início da diálise devido à aterogênese acelerada (5).

A correção dos fatores de risco não-genéticos é uma estratégia disponível para a redução da prevalência da nefropatia e retardo na evolução de pacientes com nefropatia estabelecida, dentre eles controle glicêmico, lipídico e pressórico, com inibidores da ECA ou bloqueadores dos receptores de angiotensina II, que além de anti-hipertensivos são antiproteinúricos. Gaede e cols. reduziram em $50 \%$ a progressão de micro para macroalbuminúria após 3,5 anos de seguimento com estas medidas, interferindo na progressão da nefropatia diabética (19).

Este estudo apresenta algumas limitações pelo fato de ser retrospectivo. Possíveis casos de DM podem não ter sido diagnosticados, o que subestimaria nossos dados de prevalência, dado mais preocupante ainda do ponto de vista do impacto do DM como etiologia de doença básica na população dialítica em nosso meio, assim como subestima a diferença de sobrevida entre os pacientes com DM e sem DM.

Nossos dados permitem concluir que na região Oeste do Paraná a ocorrência de DM como causa de IRCT vem aumentando em grandes proporções. Limitações no diagnóstico de DM podem ter ocorrido em pacientes que chegaram urêmicos terminais, subestimando a sua prevalência. Estratégias de diagnóstico precoce, de melhor controle metabólico do DM e da hipertensão arterial, com a participação de equipes multidisciplinares envolvendo clínicos gerais, endocrinologistas, nefrologistas, cardiologistas, enfermeiros, nutricionistas e outros profissionais da área de saúde, são extremamente necessárias para tentar conter esta emergente epidemia nos serviços de atendimento nefrológico e para reduzir a mortalidade excessiva e aumentar o acesso do diabético renal crônico ao transplante renal.

\section{REFERÊNCIAS}

1. Rychlik I, Miltenberger-Miltenyi G, Ritz E. The drama of the continuous increase in end-stage renal failure in patients with type 2 diabetes mellitus. Nephrol Dial Transplant 1998; 3:6-10.

2. Bruno RM, Gross JL. Prognostic factors in Brazilian diabetic patients starting dialysis. A 3.6-year follow-up study. J Diabetes Complications 2000;14:266-71.

3. Locatelli F, Pozzoni P, Vecchio LD. Renal replacement therapy in patients with diabetes and end-stage renal disease. J Am Soc Nephrol 2004; $15:$ S25-9.

4. Oliveira MB, Romão JE, Zatz R. End-stage renal disease in Brazil: epidemiology, prevention and treatment. Kidney Int 2005;68:S82-6.

5. Luke RG. Chronic renal failure - a vasculopathic state. N Engl J Med 1998;339:841-3.

6. Herzog CA, Ma JZ, Collins AJ. Poor long-term survival after acute myocardial infarction among patients on long-term dialysis. N Engl J Med 1998;39:799-805.

7. Medical Management of Type 2 Diabetes. Fourth Edition: American Diabetes Association. Clinical Educational Series 1998.

8. World Health Organization: Diabetes Mellitus: Report of a WHO Study Group. Geneva, World Health Org, 1985 (Tech. Rep. Series no 727). 
9. Bennet PH. Epidemiology of diabetes mellitus. In: Rifkin $\mathrm{H}$, Porte D Jr, eds. Ellenberg and Rifkin's Diabetes mellitus. New York: Elsevier, 1990. pp. 363-77.

10. Malerbi DA, Franco LJ. Multicenter study of the prevalence of diabetes mellitus and impaired glucose tolerance in the urban Brazilian population aged 30-69 yr. The Brazilian Cooperative Group on the Study of Diabetes Prevalence. Diabetes Care 1992;15:1509-16.

11. Murussi M, Baglio P, Gross JL, Silveiro SP. Risk factors for microalbuminuria and macroalbuminuria in type 2 diabetic patients. Diabetes Care 2002;25:1101-3.

12. Orchard TJ, Dorman JS, Maser RE, Becker DJ, Drash AL, Ellis $D$, et al. Prevalence of complications in IDDM by sex and duration. Pittsburg Epidemiology of Diabetes. Complications Study II. Diabetes 1990;39:1116-24.

13. Ismail N, Becker B, Strzelczyk P, Ritz E. Renal Disease and hypertension in non-insulin dependent diabetes mellitus. Kidney Int 1999;55:1-28.

14. Klein R, Klein BE, Moss SE, Cruickshanks KJ. Ten-year incidence of gross proteinuria in people with diabetes. Diabetes 1995;44:916-23.

15. Nelson RG, Bennett PH, Beck GJ, Tan M, Knowler WC, Mitch $W E$, et al. Development and progression of renal disease in Pima Indians with non-insulin-dependent diabetes mellitus. Diabetic Renal Disease Study Group. N Engl J Med 1996; 335:1636-42.
16. Hasslacher C, Ritz E, Wahl P, Michael C. Similar risk of nephropathy in patients with type I or II diabetes mellitus. Nephrol Dial Transplant 1989;4:859-63.

17. Perneger TV, Brancati FL, Whelton PK, Klag MJ. End-stage renal disease attributable to diabetes mellitus. Ann Intern Med 1994;121:912-8.

18. Ritz E, Rychlik I, Locatelli F, Halimi S. End-stage renal failure in type 2 diabetes: a medical catastrophe of worldwide dimensions. Am J Kidney Dis 1999;34:795-808.

19. Gaede P, Vedel P, Parving H-H, Pedersen O. Intensified multifactorial intervention in patients with type 2 diabetes mellitus and microalbuminuria: the Steno type 2 randomized study. Lancet 1999;353:617-22.

Endereço para correspondência:

Luis Alberto Batista Peres

Rua São Paulo 769, ap. 901

85801-020 Cascavel, PR

E-mail: peres@certto.com.br 